\title{
Pulse Design Method for 60GHz Impulse Wireless Communication System
}

\author{
Xinli Dong ${ }^{1}$, Xueyan $\mathrm{Han}^{2}$, Jingjing Wang ${ }^{2 *}$, Qiuna $\mathrm{Niu}^{2}$ and Wei $\mathrm{Shi}^{2}$ \\ ${ }^{1}$ Qingdao Branch, China United Network Communications Corporation, China \\ ${ }^{2}$ School of Information Science \& Technology, Qingdao University of Science \& \\ Technology, Qingdao, Shandong Province, China \\ dongxinli@wo.cn,564684119@qq.com,kathy1003@163.com, \\ niuqiuna@sdu.edu.cn, shiwei6670595@126.com \\ kathy1003@163.com
}

\begin{abstract}
The design of 60GHz pulse waveform is significant in the areas of Gigabit high speed wireless communication system within short distance and the accurate positioning based on $60 \mathrm{GHz}$ pulse. The pulse waveform design of $60 \mathrm{GHz}$ pulse are carried out with carrier and non-carrier separately and two methods are proposed and realized in this paper which are $60 \mathrm{GHz}$ pulse design based on Gaussian pulses and $60 \mathrm{GHz}$ pulse design based on window function of $N$ rectangular window convolution. This paper designs the 31order Gaussian derivative function pulse as $60 \mathrm{GHz}$ pulse taking the method based on the Gaussian pulse design. Compared to the other $60 \mathrm{GHz}$ pulses, 31-order Gaussian derivative function processes the less number of pulse peaks and the less pulse fluctuation which make it easy to be detected. According to the problem that the free license of $60 \mathrm{GHz}$ band is disaccord in different countries, a $60 \mathrm{GHz}$ pulse design method based on $\mathrm{N}$ rectangular window convolution is proposed. 2 degrees (the width of the rectangular window and the number of rectangular window) could be regulated to design pulse waveform makes this method flexible.
\end{abstract}

Keywords: 60GHz pulse, window function, carrier, non-carrier

\section{Introduction}

Wireless local area network (WLAN), wireless personal network (WPN) and high definition multimedia interface (HDMI) are making an urgent request for Gigabit wireless transmission technology [1-5]. At the same time indoor robot navigation and positioning, production management and other fields also have a significant demand for high-precision navigation and position technologies [6-7]. But existing wireless technologies such as ultra wide-band (UWB) and wireless fidelity (Wi-Fi) could not meet the urgent needs because of spectrum resource, transmit power and other bottleneck problems[8-12]. New technical and theoretical solutions are needed urgently. With the development of semiconductor silicon processing technology and low cost integration solutions, the $60 \mathrm{GHz}$ millimeter wave provides an effective way to solve the above problems [13]. Indoor centimeter level ranging and positioning can be realized by using the time resolution of $60 \mathrm{GHz}$ pulse, and with the advantages of free license to use several $\mathrm{GHz}$ broadband spectrum, $60 \mathrm{GHz}$ pulse technology can be described as the best choice to achieve Gigabit wireless transmission.

\footnotetext{
${ }^{*}$ Corresponding Author
} 
Compared with the traditional single carrier-frequency domain equalization (SCFDE) $60 \mathrm{GHz}$ system and orthogonal frequency division multiplexing (OFDM) $60 \mathrm{GHz}$ system, the $60 \mathrm{GHz}$ impulse wireless communication system possesses strong anti-multipath interference ability, simple circuits and low power consumption. To carry out the research on the $60 \mathrm{GHz}$ pulse wireless communication system, we must design the pulse waveform which is suitable for the $60 \mathrm{GHz}$ pulse system.

According to the technical characteristics of $60 \mathrm{GHz}$ pulse wireless communication system, the basic principle of $60 \mathrm{GHz}$ pulse design is as follows:

$\diamond$ Tens of picosecond pulses of width, or even narrower, to ensure that the spectrum occupied by a wide spectrum.

$\diamond$ The Fluctuation of waveform is small, that is, there is not much of the peak. Otherwise, the small delay will cause the matching error when the correlation detection is used which is not conducive to the detection of the receiving pulses.

$\diamond$ The DC component of the waveform is zero, so as to ensure the effective radiation of the pulse energy.

$\diamond$ Meet the $60 \mathrm{GHz}$ system spectrum masks.

$\diamond$ High frequency spectrum utilization.

$\diamond$ Easy to achieve.

$\diamond$ Easy to be detected.

$\diamond$ Can improve the system performance and achieve a lower bit error rate.

The $60 \mathrm{GHz}$ pulse waveform design method based on the inverse Fourier transform presented in [14-16] processes very high frequency spectrum utilization, but the pulse is difficult to achieve at present because of the complex waveform. The pulse design methods presented by [17-21] are interesting which are cable of being referred in designing $60 \mathrm{GHz}$ pulse.

The pulse wave design of the $60 \mathrm{GHz}$ pulse are carried out with carrier and noncarrier separately and two methods are proposed and realized in this paper which are $60 \mathrm{GHz}$ pulse design based on Gaussian pulses and $60 \mathrm{GHz}$ pulse design based on $\mathrm{N}$ rectangular window convolution. This paper designs the 31-order Gaussian derivative function pulse as $60 \mathrm{GHz}$ pulse taking the method based on the Gaussian pulse design. Compared to the other $60 \mathrm{GHz}$ pulses, 31-order Gaussian derivative function processes the less number of pulse peaks and the less pulse fluctuation which make it easy to be detected and captured. According to the problem that the free license of $60 \mathrm{GHz}$ band is disaccord in different countries, a $60 \mathrm{GHz}$ pulse design method based on $\mathrm{N}$ rectangular window convolution is proposed. And 2 degrees could be regulated to design pulse waveform makes this method flexible. This method not only can be used to design $60 \mathrm{GHz}$ pulse but also can be used to design other pulses which meet other spectrum masks by arranging the two freedegrees which possesses high flexibility and versatility.

The rest of the paper is organized as follows. $60 \mathrm{GHz}$ pulse design method based on Gaussian pulses is presented in Section 2. Section 3 provides the $60 \mathrm{GHz}$ pulse design based on $\mathrm{N}$ rectangular window convolution. Concluding remarks are given in Section 4. 


\section{60GHz Pulse Design Method based on Gaussian Pulses}

Gaussian pulse and its derivative pulses can be expressed as follows.

$$
\begin{aligned}
& g_{0}(t)=A e^{-\frac{2 \pi t^{2}}{\alpha^{2}}}, \\
& g_{1}(t)=A\left(-\frac{4 \pi t}{\alpha^{2}}\right) e^{-\frac{2 \pi t^{2}}{\alpha^{2}}}, \\
& g_{2}(t)=A \frac{4 \pi}{\alpha^{4}} e^{-\frac{2 \pi t^{2}}{\alpha^{2}}}\left[-\alpha^{2}+4 \pi t^{2}\right], \\
& g_{3}(t)=A \frac{(4 \pi)^{2}}{\alpha^{6}} t e^{-\frac{2 \pi t^{2}}{\alpha^{2}}}\left[3 \alpha^{2}-4 \pi t^{2}\right],
\end{aligned}
$$

where $g_{0}(t)$ is Gaussian pulse, $g_{j}(t)$ is its jth derivative $(\mathrm{j}>1)$, A is used to normalize the pulse amplitude, $\alpha$ is the pulse shape factor. The bigger the $\alpha$, the wider the pulse, and corresponding the narrower the frequency band.

The Power Spectral Density (PSD) of pulse $\mathrm{g}(\mathrm{t})$ is given by $P_{g}(f)$.

$P_{g}(f)=\frac{1}{T}|G(f)|^{2}$

where $G(f)$ is the Fourier transform of pulse $g(t)$. The Fourier transform of Gaussian series pulses are

$G_{k}(f)= \pm A \frac{\alpha}{\sqrt{2}} e^{-\frac{\pi \alpha^{2} f^{2}}{2}}(j 2 \pi f)^{k}, \quad k=0,1,2 \cdots$.

The PSD of Gaussian series pulses can be expressed as

$P_{g}(f)=\frac{A^{2} \alpha^{2}}{2 T_{p}} e^{-\pi \alpha^{2} f^{2}}(2 \pi f)^{2 k}, k=0,1,2 \cdots$

By formula (3), the Fourier transform of Gaussian pulse Kth derivative possesses the properties:

$G_{k}{ }^{\prime}(f) \propto f^{k} e^{-\frac{\pi \alpha^{2} f^{2}}{2}}, k=0,1,2 \cdots$.

Differentiate the right part of formula (4) and make it zero. The relationship of the peak frequency $f_{\text {peak }}$, the order $\mathrm{k}$ and the pulse shape factor $\alpha$ can be obtained as

$f_{\text {peak }}=\sqrt{k} \frac{1}{\alpha \sqrt{\pi}}$

Formula (5) shows two points.

The peak frequency increases with the increase of the order number of Gaussian function. In other words, the energy is shifted to higher frequency by differential.

The peak frequency $f_{\text {peak }}$ increases with the decrease of the pulse shape factor $\alpha$. 


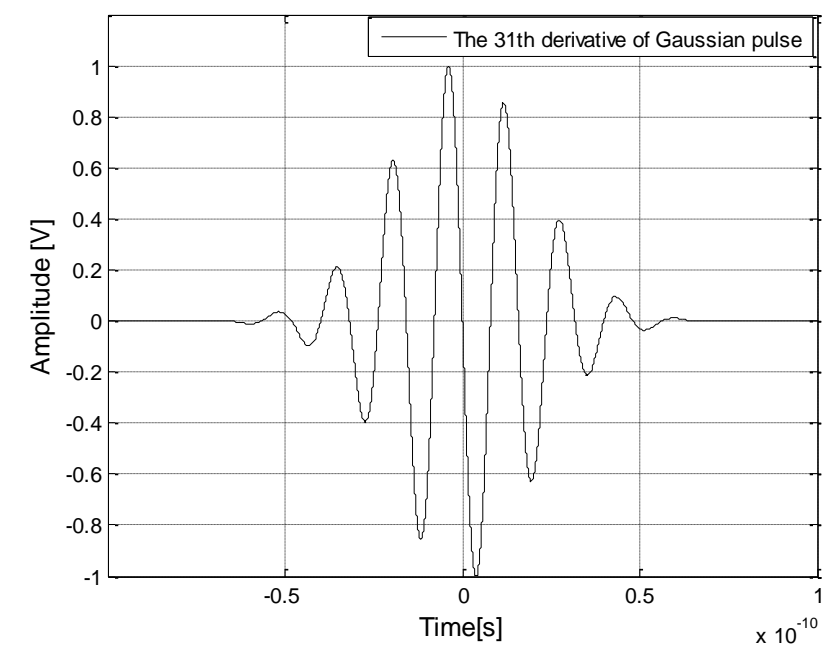

Figure 1. 60GHz Pulse Waveform based on the 31 Order Derivative of Gaussian

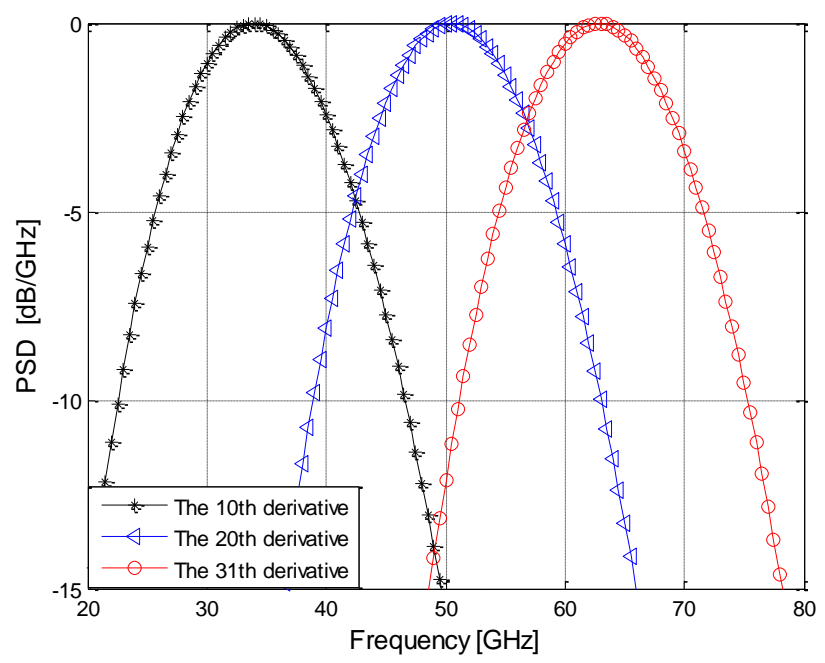

Figure 2. PSD of $60 \mathrm{GHz}$ Pulse based on the 31 Order Derivative of Gaussian

The pulse waveform and its PDF of $60 \mathrm{GHz}$ based on the 31 order derivative of Gaussian are shown on Figure 1, and Figure 2, with the pulse shape factor $\alpha=0.036 \mathrm{~ns}$. The center frequency of $60 \mathrm{GHz}$ pulse is $60.5 \mathrm{GHz}$.

Although many differential increases the difficulty, but the number of peaks in the Gaussian 31 order derivative pulse is less, the pulse fluctuation is small which is favorable for the detection and capture of the received signal.

\section{60GHz Pulse Design based on N Rectangular Window Convolution}

The According to the problem that the free license of $60 \mathrm{GHz}$ band is disaccord in different countries, a $60 \mathrm{GHz}$ pulse design method based on window function of $\mathrm{N}$ rectangular window convolution is proposed. Because 2 degrees (the width of the rectangular window and the number of rectangular window) could be regulated when using this method to design pulse waveform, this method is flexible. This method not only can be used to design $60 \mathrm{GHz}$ pulse but also can be used to design 
other pulses which meet other spectrum masks by arranging the two free-degrees which possesses high flexibility and versatility.

\subsection{Design Idea}

For a given spectrum mask, the center frequency $f_{c}$ of the spectrum mask should be determined at first. Add a window function to the cosine function $f(t)=\cos \left(2 \pi f_{c} t\right)$ then a quasi pulse described as formula (6) can be obtained.

$p(t)=f(t) \times w\left(\frac{t}{\tau}\right)$

where $p(t)$ is the $60 \mathrm{GHz}$ pulse to be designed, it is a time limited pulse, and $w\left(\frac{t}{\tau}\right)$ is the window function. The parameter $\tau$ is the time length of window function and also the pulse duration of $p(t)$.

There are a lot of options for window function $w(t)$. Consider the simplest window functions for rectangular windows, as shown in the formula (7).

$$
w_{1}(x)=\left\{\begin{array}{l}
1|x|<\frac{1}{2} \\
\frac{1}{2}|x|=\frac{1}{2} \\
0|x|>\frac{1}{2}
\end{array}\right.
$$

Take the rectangular window function to the formula (6), limit the time of cosine function to $\left(-\frac{\tau}{2}, \frac{\tau}{2}\right)$, and the pulse $p(t)$ detained is the time limit for pulse duration time $\tau$. Fourier transform the formula (7) to obtain the spectrum of the rectangular window, as formula (8) shown.

$W_{1}(f)=\tau \sin c(f \tau)$

where the function $\sin c($.$) is defined as follows.$

$\sin c(x)= \begin{cases}\frac{\sin (\pi x)}{\pi x} & \text { if } x \neq 0 \\ 1 & \text { if } x=0\end{cases}$

The pulse obtained by using the rectangular window and formula (6) is expressed as $p_{1}^{e}(t)$. The spectrum of $p_{1}^{e}(t)$ can be obtained by $w_{1}(t / \tau)$ and the convolution of the spectrum of $f(t)$, as shown in formula (10).

$$
\begin{aligned}
P_{1}^{e}(f) & =\frac{1}{2}\left[\delta\left(f-f_{c}\right)+\delta\left(f+f_{c}\right)\right] * W_{1}(f) \\
& =\frac{\tau}{2}\left\{\sin c\left[\tau\left(f-f_{c}\right)\right]+\sin c\left[\tau\left(f+f_{c}\right)\right]\right\}
\end{aligned}
$$

where $*$ stands for the convolution operation.

Here are two questions to be solved.

1) The spectrum of the $60 \mathrm{GHz}$ pulse is designed to meet the requirements of the frequency mask.

2) The $60 \mathrm{GHz}$ pulse designed has no DC component. 
To the second question, this limit make the choice of window function $w(t / \tau)$ more complicated, so we try to avoid this difficulty. For this, the following regulations are put forward as: when the rectangular window $w_{1}(t / \tau)$ shown in formula (7) is used, the pulse $p_{1}^{e}(t)$ has no DC component if and only if $\tau_{c}=k, k=1,2, \ldots \ldots$

The rules are derived from the following reasons: the period of $f(t)=\cos \left(2 \pi f_{c} t\right)$ is $1 / f_{c}$, and the period of $w_{1}(t / \tau)$ is $\tau$ ( in other words, the width of $w_{1}(t / \tau)$ is $\tau$ ), that means $f(t)$ is measured by $\tau . \tau=k \cdot \frac{1}{f_{c}}$ is to make sure integer cosine function $f(t)$ which ensure that the $60 \mathrm{GHz}$ pulse designed should have no DC component.

Now consider a more general situation. The window function $w_{n}(t / \tau)$ generated by convolving $\mathrm{n}$ rectangular windows $w_{1}(t / \tau)$, is defined as

$w_{n}\left(\frac{t}{\tau}\right)=\underbrace{w_{1}\left(\frac{t}{\tau}\right) * w_{1}\left(\frac{t}{\tau}\right) * \cdots w_{1}\left(\frac{t}{\tau}\right)}_{n}$

where $n$ is the number of rectangular windows convolved.

The window function $w_{n}(t / \tau)$ is obtained by convolving $\mathrm{n}$ rectangular windows $w_{1}(t / \tau)$, and its width is $n \tau$, its Time domain expression is shown as formula (12).

$$
\begin{aligned}
w_{n}\left(\frac{t}{\tau}\right)= & \sum_{m=0}^{n} \frac{n\left[\frac{t}{\tau}-\left(\frac{n}{2}-m\right)\right]^{n-1}(-1)^{n-m}}{m !(n-m) !} \\
& \times U\left[\frac{t}{\tau}-\left(\frac{n}{2}-m\right)\right]
\end{aligned}
$$

where $U[\cdot]$ is the unit step function.

If window function $w_{n}(t / \tau)$ is used in formula (6), the duration time of pulse $p_{n}^{e}(t)$ is $n \tau$. The frequency spectrum of $w_{n}(t / \tau)$, denoted as $W_{n}(f)$, is given by

$W_{n}(f)=W_{1}^{n}(f)=\tau^{n} \sin c^{n}(\tau f), \quad n=1,2,3, \ldots \ldots$

So, the frequency spectrum of $p_{n}^{e}(t)$ can be donated by $P_{n}^{e}(f)$ as

$$
P_{n}^{e}(f)=\frac{\tau^{n}}{2}\left\{\sin c^{n}\left[\tau\left(f-f_{c}\right)\right]+\sin c^{n}\left[\tau\left(f+f_{c}\right)\right]\right\}
$$

Its time domain signal is

$$
p_{n}^{e}(t)=w_{n}\left(\frac{t}{\tau}\right) \cdot \cos \left(2 \pi f_{c} t\right)
$$

Observe from (10) that the pulse spectrum remains centered at $\mathrm{fc}$ and that as $\mathrm{n}$ increases the relative amplitudes of the side lobes decrease.

From formula (16), we can see that the center frequency of the pulse is kept at $f_{c}$ , and the relative amplitude of the side lobe is smaller and the width of the main lobe is narrower with the increase of $n$. 
Therefore, increasing the value of $n$ is a strategy to make the pulse spectrum fit for some spectrum mask. In fact, for a known center frequency $f_{c}$, the spectrum calculated by formula (16) possesses two degrees of freedom, $\tau$ and $n$. These two parameters are used to adjust the pulse waveform and pulse duration in the time domain, and to make sure the pulse spectrum fit for the spectrum mask in the frequency domain.

From the formula (15): the DC component of $p_{n}^{e}(t)$ is 0 for all integer values of $n$ if it is zero for $n=1$. So, once the value of $\tau$ meet the formula (11), $n$ can be any change, and the DC component of $p_{n}^{e}(t)$ is zero. A series of new pulse close to the spectrum mask can be generated by adjusting the values of $\tau$ and $n$.

Note that: in the case of all the pulses meet the spectrum mask, a pulse with short length is desirable because the longer pulse duration will lead to greater multi-user interference.

A longer pulse duration is undesirable in multiple access environments because it leads to greater multiple access interference.

The design idea based on $\mathrm{N}$ rectangular window convolution can be further expanded, and the other windows can be obtained by convoluting the basic window repeatedly. For example, a certain pulse fit for some spectrum mask can also be obtained by convoluting $n$ finite time long Gaussian pulse.

\subsection{Design Process}

We take the spectrum mask regulated by Federal Communications Commission (FCC) for $60 \mathrm{GHz}$ wireless communication system and design $60 \mathrm{GHz}$ pulses based on $\mathrm{N}$ rectangular window convolution. Assuming that the spectral mask is normalized, the center frequency of the spectrum mask is set to $60.5 \mathrm{GHz}$.

The main idea of this method is: adjust the parameter $\tau$ and $n$ simultaneously, then many pulse meet the spectrum mask regulated by FCC for $60 \mathrm{GHz}$ wireless communication system can be obtained, but we should choose the pulse with smallest value of $n \tau$ to avoid the influence of multi-user interference brought by long pulse duration time $n \tau$.

Specific steps are as follows:

1) Set $f_{c}$

Set $f_{c}$ according spectrum mask. The period of cosine carrier $f(t)$, with the value of $1 / f_{c}$, appeared in formula (6) is set actually when $f_{c}$ is set.

2) Set $\tau$

$\tau$ is the period of rectangle window $w_{1}(t / \tau)$. By formula (11) we obtain: $\tau=k \cdot \frac{1}{f_{c}}$, where $k$ must be taken an integer. The meaning of $\tau=k \cdot \frac{1}{f_{c}}$ is there are $k$ periods of cosine carrier $f(t)$ in one period of rectangle window $w_{1}(t / \tau)$. Therefore, after $f_{c}$ is set, setting set $\tau$ means setting $k$ appeared in formula (11).

3) Set $n$

When $k=8$ and $n=1, \tau=k \cdot \frac{1}{f_{c}}=0.13223 n s$, the PSD of $60 \mathrm{GHz}$ pulse, denoted by $p_{1}(t)$, designed based on $\mathrm{N}$ rectangular window convolution is shown in Figure 3. Obviously, $p_{1}(t)$ cannot meet the spectrum mask regulated by FCC for $60 \mathrm{GHz}$ communication system perfectly, and it should be adjusted. According to the design idea of $\mathrm{N}$ rectangular window convolution, we should increase $n$. 


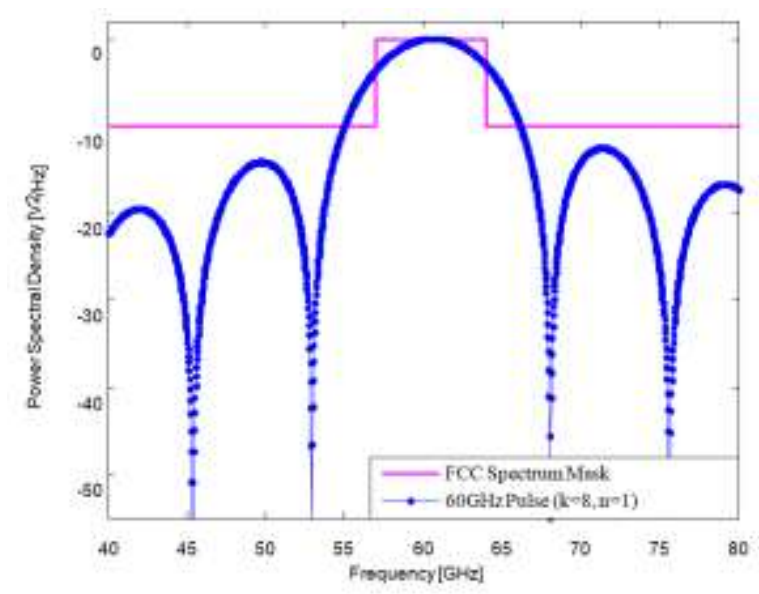

Figure 3. PSD of $60 \mathrm{GHz}$ Pulse $p_{1}(t)$ based on Rectangular Window Convolution

We set $n=2, k=8(\tau=0.13223 n s)$, and the PSD of 60GHz pulse, denoted by $p_{2}(t)$, designed based on $\mathrm{N}$ rectangular window convolution is shown in Figure 4. Obviously, $p_{2}(t)$ still cannot meet the spectrum mask regulated by FCC for $60 \mathrm{GHz}$ communication system, and it should be adjusted too.

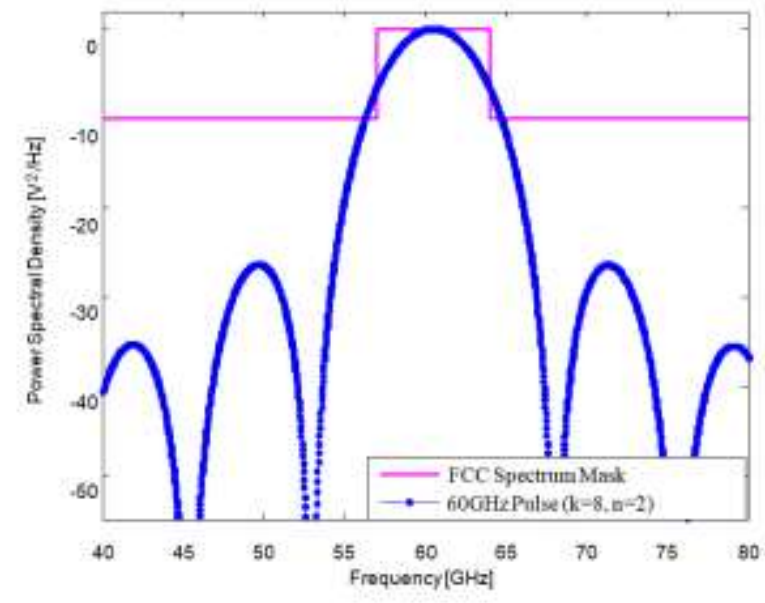

Figure 4. PSD of $60 \mathrm{GHz}$ Pulse $p_{2}(t)$ based on Rectangular Window Convolution

But we can see from Figure 3, and Figure 4, that the PSD of $p_{2}(t)$ obtained when $n=2, k=8$ is closer to frequency mask than $p_{1}(t)$ obtained when $n=1$, $k=8$. And we can see from Figure 3, and Figure 4, that the center frequency of pulse is maintained at $f_{c}$, while the relative amplitude of the side lobe is smaller and the width of the main lobe is narrower with the increase of $n$. Therefore, increasing the value of $n$ is a kind of strategy which can meet the specific spectrum mask. In accordance with the design idea of rectangular window convolution, the value of $n$ should be increased continuously.

We set $n=3, k=8(\tau=0.13223 n s)$, and the PSD of $60 \mathrm{GHz}$ pulse, denoted by $p_{3}(t)$, designed based on $\mathrm{N}$ rectangular window convolution is shown in Figure 
$5, p_{3}(t)$ is cable of fitting for the spectrum mask regulated by $\mathrm{FCC}$ for $60 \mathrm{GHz}$ communication system.

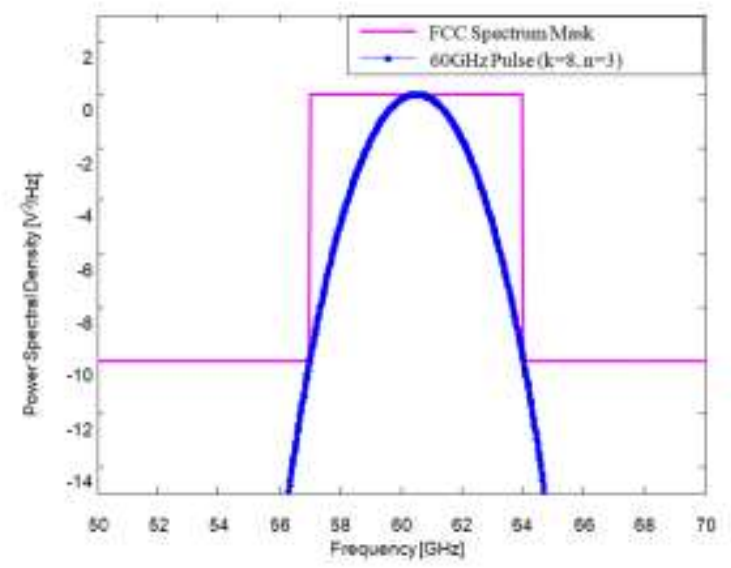

Figure 5. PSD of 60GHz Pulse $p_{3}(t)$ based on Rectangular Window Convolution

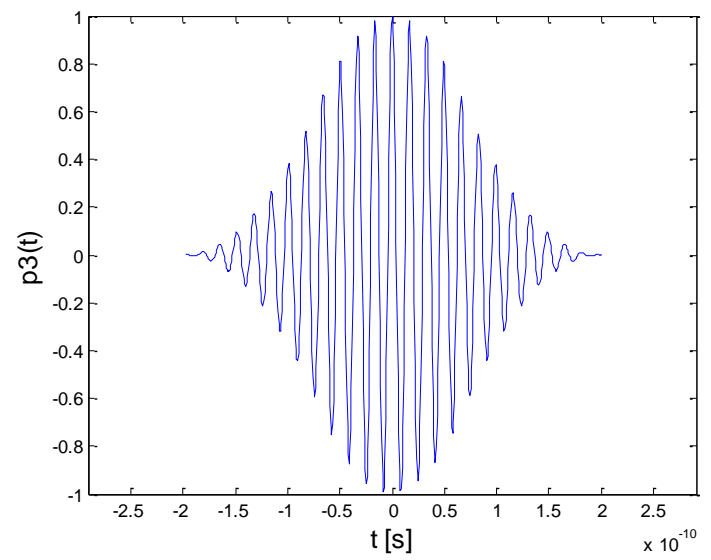

Figure 6. Time Domain Waveform of $60 \mathrm{GHz}$ Pulse $p_{3}(t)$ based on Rectangular Window Convolution

Time domain waveform of $p_{3}(t)$ is shown in Figure 6 , where the amplitude is normalized, the pulse length is $0.4 \mathrm{~ns}$.

The $60 \mathrm{GHz}$ pulse design method based on $\mathrm{N}$ rectangular window convolution is more flexible than the method based on Gaussian pulse. For the design method based on the Gaussian pulse, the center frequency and the bandwidth of the spectrum are all determined by the pulse shape factor $\alpha$. $\alpha$ can be used to set the pulse width and influence the center frequency simultaneously. The center frequency increases with the decrease of the pulse shape factor $\alpha$, so we can only move the frequency spectrum to the $60 \mathrm{G}$ band by decreasing $\alpha$. However, decreasing $\alpha$ will increase the frequency spectrum of the pulse which make it unable to meet the spectral mask.

Different from the method based on the Gaussian pulse, the center frequency of the pulse frequency spectrum can be arbitrarily set according to the $60 \mathrm{GHz}$ pulse design method based on $\mathrm{N}$ rectangular window convolution. Moreover, the proposed method can provide two degrees of freedom $\tau$ and $n$ which can be used to adjust the pulse waveform to meet a given spectrum mask and a lot of spectrum mask 
pulses can be obtained by setting the appropriate combination of the values of $\tau$ and $n$.

The most significant is that this method not only can be used to design $60 \mathrm{GHz}$ pulse but also can be used to design other pulses which meet other spectrum masks by arranging the two free-degrees which possesses high flexibility and versatility.

\section{Conclusion}

Designing the $60 \mathrm{GHz}$ pulse waveform is significant to achieve $60 \mathrm{GHz}$ impulse wireless communication system which possesses strong anti-multipath interference ability, low power consumption and other advantages. Two pulse waveform design methods of the $60 \mathrm{GHz}$ pulse are presented and realized in this paper. One utilizes the 31order Gaussian derivative function pulse as $60 \mathrm{GHz}$ pulse which processes the less number of pulse peaks and the less pulse fluctuation which make it easy to be detected. Another exploits $\mathrm{N}$ rectangular window convolution which provides 2 degrees that possesses high flexibility and versatility.

\section{Acknowledgements}

The authors would like to thank the referees and editors for providing very helpful comments and suggestions.

This project was supported by the Key Laboratory of Universal Wireless Communications (Beijing University of Posts and Telecommunications), Ministry of Education, P. R. China (No. KFKT-2013102), the National Natural Science Foundation of China (No. 61304222), the China Postdoctoral Science Foundation (No. 2014M551905), the Natural Science Foundation of Shandong Province (No. ZR2012FQ021), the Open Project of State Key Laboratory of Millimeter Waves (No. K201321), and the Shandong Province Postdoctoral Special Funds for Innovation Projects (No. 128761).

\section{References}

[1] S. K. Yong, and C.-C. Chong, "An overview of multigigabit wireless through millimeter wave technology: Potentials and technical challenges," EURASIP Journal on Wireless Communications and Networking, vol. 2007, (2007), pp. 1-10.

[2]H. Zhang, X. Chu, W. Guo and S. Wang, "Coexistence of Wi-Fi and Heterogeneous Small Cell Networks Sharing Unlicensed Spectrum," IEEE Communications Magazine, vol. 53, no. 3, (2015), pp. 158-164.

[3] R. Daniels and R. Heath, Jr., "60 GHz Wireless Communications: Emerging Requirements and Design Recommendations,” IEEE Vehicular Technology Magazine, vol.2, no. 3, (2007), pp. 41-50.

[4] N. Guo, R. C. Qiu, S. S. Mo and K. Takahashi, "60 GHz millimeter-wave radio: Principle, technology, and new results," EURASIP Journal on Wireless Communications and Networking, vol. 2007, (2007), pp. $1-8$.

[5]H. Zhang, C. Jiang, N. Beaulieu, X. Chu, X. Wen and M. Tao, "Resource Allocation in Spectrum-Sharing OFDMA Femtocells with Heterogeneous Services, IEEE Transactions on Communications, vol. 62, no. 7, (2014), pp. 2366-2377.

[6]H. Liu, H. Darabi, P. Banerjee and J. Liu, "Survey of Wireless Indoor Positioning Techniques and System," IEEE Transactions on Systems, Man, and Cybernetics-Part C: Application and reviews, vol.37, no. 6, (2007), pp. 1067-1080.

[7] S. Gonzalo, A. Jose, D. Bano and G. Risueno, "Challenges in Indoor Global Navigation Satellite Systems," IEEE Signal Processing Magazine, vol. 29, no.2, (2012), pp. 108-131.

[8] M. Gunawan, B. Li, T. Gallagher, A. Dempster, and G. Retscher, "A new method to generate and maintain a WiFi fingerprinting database automatically by using RFID," International Conference on Indoor Positioning and Indoor Navigation, (2012), pp. 1-6.

[9]C. Lim, Y. Wan, B. Ng and C. Samson, "A Real-Time Indoor WiFi Localization System Utilizing Smart Antennas,” IEEE Transactions Consumer Electronics, vol.53, no.2, (2013), pp.618-622.

[10] J. Li, J. Fang and S. Ge, "Kinetics and Design of a Mechanically Dithered Ring Laser Gyroscope Position and Orientation System," IEEE Transactions on Instrumentation and Measurement, vol. 62, no.1, (2013), pp. 210-220.

[11] G. Garcia, L. Muppirisetty and H. Wymeersch, "On the Trade-off between Accuracy and Delay in UWB Navigation,” IEEE Communications Letters, vol. 17, no. 1, (2013), pp. 39-42. 
[12] S. Wang, J. An, Y. Ren, T. Wang and X. Bu, "Compressed Receiver for Multipath DSSS Signals," IEEE Communications Letters, vol. 18, no. 8, (2014), pp. 1359-1362.

[13] J. Wang, X. Liang, W. Shi, Q. Niu, T. Aaron Gulliver and X. Liu, "Fingerprinting Localization based on $60 \mathrm{GHz}$ Impulse Radio," 2011 IEEE Pacific Rim Conference on Communications, Computers and Signal Processing, Victoria, BC, Canada, (2015), August 24-26.

[14] H. Zhang, T. Lu and T. A. Gulliver, "Pulse waveforms for $60 \mathrm{GHz}$ M-ary pulse position modulation communication systems," IET Communications, vol.7, no.2, (2013), pp. 169-179.

[15] J. Wang, "Study on Channel Capacity and Performance of 60GHz Wireless Communication Systems," Doctoral Dissertation, (2012), The Ocean University of China.

[16] H. Zhang, T. Lu, J. Wang and A. Gulliver, "Pulse waveforms for $60 \mathrm{GHz}$ impulse radio communications," 2011 IEEE Pacific Rim Conference on Communications, Computers and Signal Processing, Victoria, BC, Canada, (2011), August 23-25.

[17] B. Parr, B. Cho, K. Wallace, and Z. Ding, "A novel ultra-wideband pulse design algorithm," IEEE Communications Letters, vol. 7, no. 5, (2003), pp. 219-221.

[18] N. Beaulieu and B. Hu, "A novel pulse design algorithm for ultra-wideband communications," in the Conference of IEEE GLOBECOM, Dallas, Texas USA, (2004) Novemeber, pp. 3220-3224.

[19] Z. Bai, X. Li,D. Yuan and K. Kwak, "Non-linear chirp based UWB waveform design for suppression of NBI," Wireless Communications and Mobile Computing, vol. 12, no. 6, (2012), pp. 545-552.

[20] W. Zou, C. Zhang, and Z. Zhou, "Algorithm to design UWB pulse based on the peak frequency," Journal on Communications, vol. 26, no. 9, (2005), pp. 74-78.

[21] Z. Bai, J. Liu, H. Chen, "Design of ultra-wideband pulses based on spectrum shifted Gaussian waveforms," Institution of Engineering and Technology, vol. 7, no. 6, (2013), pp. 512-520.

\section{Authors}

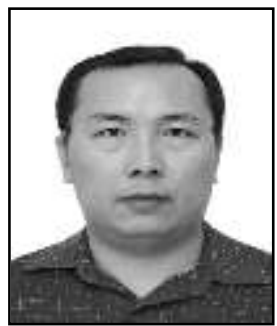

Xinli Dong, received his B.S. degree in Mechanical Engineering from China University of Petroleum, P.R. China in 1997, the M.Sc. degree in Computer Application Technology, Ocean University of China, P.R. China in 2005. From 2005 to now, he is an engineer in Qingdao Branch, China United Network Communications Corporation. His research interests include MIMO wireless systems, M2M wireless communications and cooperative communication networks.

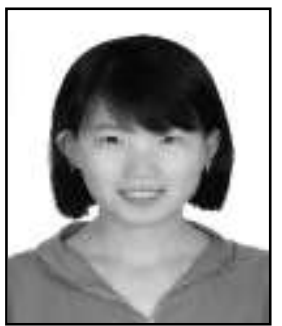

Xueyan Han, received her B.S. degree in Electronic Information Science and Technology from Qingdao University of Science \& Technology, Qingdao, China, in 2013. From 2014 to now, she is a graduate student at the College of Information Science \& Technology, Qingdao University of Science \& Technology. Her research interests include ultra wideband radio systems and $60 \mathrm{GHz}$ wireless position technology.

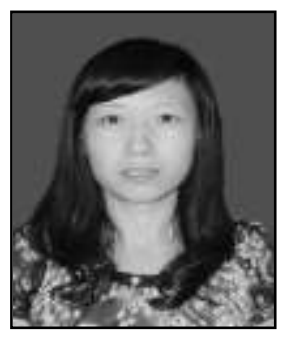

Jingjing Wang, received her B.S. degree in Industrial Automation from Shandong University, Jinan, China, in 1997, the M.Sc. degree in Control Theory and Control Engineering, Qingdao University of Science and Technology, Qingdao, China, in 2002, and the Ph.D. degree in Computer Application Technology, Ocean University of China, Qingdao, China, in 2012. From 2002 to now, she is an associate professor at the College of Information Science \& Technology, Qingdao University of Science \& Technology. From 2014 to now, she is a visiting professor in the University of British Columbia. Her research interests include $60 \mathrm{GHz}$ wireless communication, $60 \mathrm{GHz}$ wireless position technology, ultra wideband radio systems, MIMO wireless communications, M2M wireless communications and cooperative communication networks. 


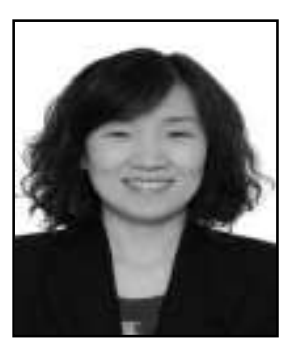

Qiuna Niu, has completed her Bachelor of industrial automation in Changchun University of Science and Technology, Changchun, China in 2001, and has her M.S in Communication and Information System from Shandong University, Jinan, China 2004. She is a Ph.D. candidate of Ocean University of China. From 2004 to now, she is a lecturer at the College of information Science \& Technology, Qingdao University of Science \& Technology. Her current research interests include information theory and coding, wireless communication and networks.

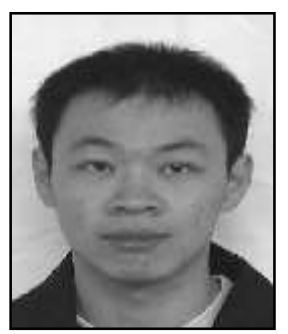

Wei Shi, received his B.S. degree in Ludong University, Yantai, China, in 2009, and the Master Degree in Signal and Information processing from Ocean University of China, Qingdao, China in 2011, and the Ph.D. degree in Computer Application Technology, Ocean University of China, Qingdao, China, in 2014. From 2014 to now, he is a lecturer in College of Information Science \& Technology, Qingdao University of Science \& Technology. His research interests include OFDM, MIMO, LTE, UWB, $60 \mathrm{GHz}$ wireless communication, cooperative communication networks and indoor positioning. 